\title{
From drifting polyaniline sensor to accurate sensor array for breath analysis
}

\author{
P. Le Maout*, J-L. Wojkiewicz ${ }^{\dagger}$, N. Redon ${ }^{\dagger}$, C. Lahuec*, F. Seguin*, L. Dupont, A. Pud ${ }^{\ddagger}$ and S. Mikhaylov \\ ${ }^{*}$ Elec. Dept, Optics Dept.* IMT Atlantique, Technopole Brest Iroise, BREST, France \\ Email: paul.lemaout@imt-atlantique.fr \\ $†$ IMT Lille Douai, Univ. Lille, SAGE-Dept F-59000 Lille, France \\ ¥Institute of Bioorganic Chemistry and Petrochemistry, National Academy of Science of Ukraine
}

\begin{abstract}
Kidney failure is a critical chronic disease, defined as the irreversible loss of kidney functions. It has been shown that this pathology is associated with an increase of ammonia concentration in breath. Measuring it with a handheld system is a simple way for a noninvasive and early diagnostic. The idea of this paper is to measure the concentration of ammonia in a concentration range of human breath $(500 \mathrm{ppb}-2100 \mathrm{ppb})$ with humidity using a network of 11 different nanocomposite sensors. To overcome sensor weaknesses (sensor drift and sensitivity to humidity), the electronic nose principles are applied. Polyanilinebased nanocomposites with titanium dioxide, chitosan and carbon nanotubes are used to provide different sensitivities and response times and thus associate a single pattern to a concentration range. Several classifiers are then investigated and recursive feature elimination algorithm are used to select the most relevant features and sensors while improving the measurement accuracy. Measurerement accuracy reaches $91 \%$ with the combination of feature selection and Support Vector Machine algorithm.
\end{abstract}

\section{INTRODUCTION}

Human breath is composed of several hundred compounds with a high relative humidity, above $90 \%$ [1]. In recent years, the correlation between diseases and gas compounds present in the breath has been studied [2]. For instance, ammonia is one of the most relevant markers for hepatic or kidney diseases [3]. This compound is a protein breakdown product which is normally converted into urea by the liver and excreted by the kidneys. In the case of failure of one of these two organs, the ammonia concentration increases in exhaled breath from a few hundred ppb when healthy up to several ppm [4]. In this study, up to $1.1 \mathrm{ppm}$ of ammonia is classified as healthy, and above $1.6 \mathrm{ppm}$ as unhealthy [5]. Because the healthy/unhealthy boundary is blurred, an intermediate concentration range is specified.

To achieve an economically sustainable system to be provided to general practitioners, autonomous, handheld and user friendly, measurement systems based on a single gas sensor or gas sensor array are a promising solution. However, limitations related to detection limit, non-specificity, response time and humidity sensitivity must be taken into account.

Using the electronic nose principle is the proposed solution to solve sensors issues and meet the specifications of the

This work was supported by Institut Mines Telecom Fundation in the "Future et Ruptures" program. application : this means associating a single print of sensor responses with a concentration range by using classification algorithms [6]. It therefore requires sensors with differentiated responses. It becomes possible to reduce drift, aberrant values while maintaining the diagnostic accuracy. Polyaniline is chosen to form the sensitive base material for the sensor array because it gives many advantages: sensitive to ammonia, low cost, and it is possible to make different formulations to have differentiated responses.

The aim is to determine the optimal combination of surface compositions to use, the best characteristics of the curve responses and the best classification algorithm. The rest of this paper is organized as follows: the first part tackles the fabrication of the sensors, the sensing system and the extraction of the features and algorithms that are used. The last part deals with the results and the conclusion.

\section{EXPERIMENTAL}

\section{A. Sensor fabrication}

A polyaniline-based formulation is deposited on interdigitated chrome/gold electrodes on each sensor. A total of 11 sensors are fabricated. The synthesis of different polyaniline nanocomposites are explained below. The first type is based on chitosan, a biopolymer derived from crustacean shell. It is a non-toxic and stable material. Co-dissolution is the method used to synthesize this first nanocomposite. Previous studies showed responses down to $200 \mathrm{ppb}$ of ammonia but with hydrophilic properties [7]. All polyaniline sensors are both sensitive to ammonia and humidity. The latter parameter affects much more the response of the sensors than ammonia because water is at a higher concentration in the breath. It is then important to have sensors with different sensitivities to humidity in order to separate the contribution of moisture from ammonia by classification. The second nanocomposite is based on titanium dioxide, $\mathrm{TiO}_{2}$, a semi-conducting metal oxide nanoparticle. Polyaniline coats the $\mathrm{TiO}_{2}$ particle, giving a high surface/volume ratio giving a very low detection limits of about 50 ppt. The complete synthesis process and characterization are described in [8]. Finally, a synthesis of polyaniline with carbon nanotubes (CNTs) is used. The electrical resistance of this synthesis is thus function of the polyaniline and the electrical paths made by the CNTs. Sensors 


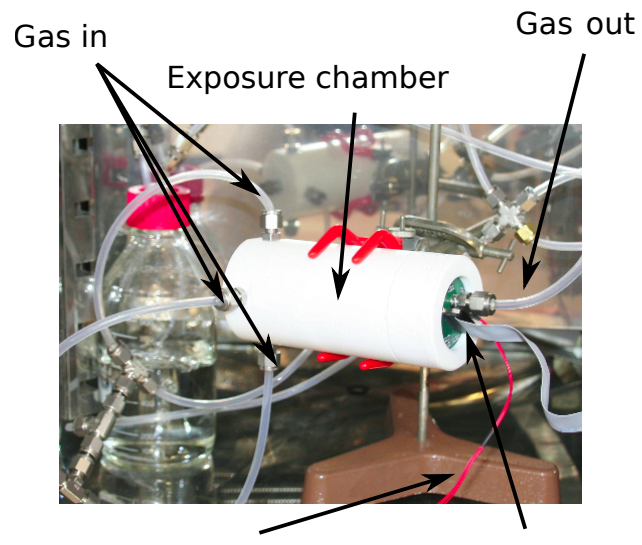

Probe $\mathrm{T}^{\circ} \mathrm{C}$ and $\mathrm{RH}$ Sensors to multimeter

Fig. 1. Picture of the sensor array test bench. Mass Flow Controller (MFC) controls the individual mixing channels to the exposure chamber.

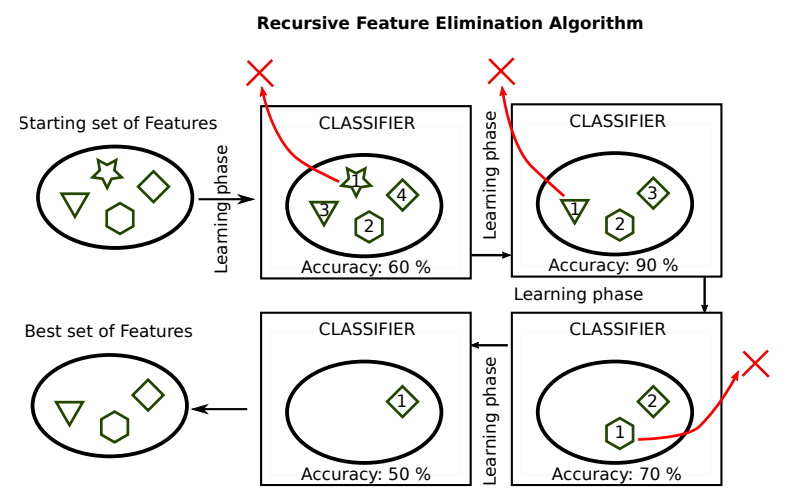

Fig. 2. Exemple of Recursive Feature Elimination algorithm with a starting set of 4 features. The best subset is the one with the best classification accuracy.

$1,2,6$ and 7 are based on chitosan, sensors 3,4,8,9,10 and 11 are based on $\mathrm{TiO}_{2}$ and sensor 5 is based on CNTs.

\section{B. Sensing system}

The sensor array is then placed in an exposure chamber. Moisted air and ammonia are mixed and injected for 1 minute into the exposure chamber, Figure 1 . The amount of ammonia added in the mixture varies from $500 \mathrm{ppb}$ to $2 \mathrm{ppm}$. Three classes are defined: Healthy, Uncertain, and Unhealthy. They correspond to three concentration ranges of ammonia : respectively $500 \mathrm{ppb}-1.1 \mathrm{ppm}, 1.1 \mathrm{ppm}-1.7 \mathrm{ppm}, 1.7 \mathrm{ppm}$ $2.8 \mathrm{ppm}$. The exposure chamber is in a climatic room, with a controlled temperature of $37^{\circ} \mathrm{C}$. The flow rate is at $8 \mathrm{~L}^{\mathrm{min}}{ }^{-1}$. After an exposure, a purified and dry air is injected during 1 minute. A total of 250 cycles are then realised with no specific order in the concentration of ammonia added in the mixture.

\section{Feature extraction and selection}

Typically, only the maximum variation is extracted from the curve. However, it has been shown that transient features such as integrals and/or derivative could also help increasing the classification performance [9]. The set of features extracted is thus:

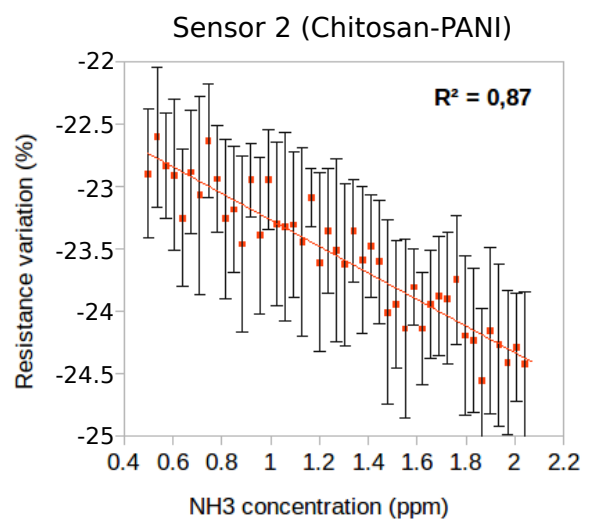

Fig. 3. Resistance variation versus ammonia concentration for sensor 2 based on chitosan.

- the maximum derivative of the signal: $\delta_{R}$,

- the integral value: $\int R$,

- the maximum variation: $\Delta_{R}$.

For each sensor, features are extracted during both the absorption and desorption phases, yielding a total of 66 features (six extracted features for the eleven sensors). After extracting the features, it is useful to select the best subset of features as it provides a more accurate classification result.

If $n$ is the feature number, $2^{n}-1$ training calculations are needed to find the best subset with an exhaustive search. In this study, $n$ is 66, it is unrealistic to find an optimal subset of features. Figure 2 shows how the features are then selected with the Recursive Feature Elimination algorithm. Several classification algorithms are investigated: Discriminant Analysis (LDA), Random Forest (RF), Support Vector Machine (SVM) and Multi-Layer Perceptron (MLP) [10].

\section{RESULTS}

\section{A. Sensing properties}

Figure 3 shows the maximum variation of the resistance with ammonia concentration for sensor 2, based on chitosan. As expected, chitosan sensors have a much higher relative resistance variation, around $23 \%$ compared to $\mathrm{TiO}_{2}$ sensors, around $3 \%$. Humidity affects sensor 2 more because of the hydrophilic properties of chitosan. However, resistance decreases for sensor 2 and increases for sensor 4 , based on $\mathrm{TiO}_{2}$. The explanation of these different responses is that humidity and ammonia interact in different ways with these nanocomposites. The ammonia deprotonates the polyaniline. As a consequence, the resistance increases while humidity inflates the matrix of the sensors. This inflation increases the number of paths for the electrons, so the resistance decreases. As chitosan is hydrophilic, the inflation effect dominates.

Within the measured concentration range, chitosan and $\mathrm{TiO}_{2}$ sensors both have linear responses. However, the $R^{2}$ coefficient is better for $\mathrm{TiO}_{2}$ sensors, 0.94 compared to 0.87 for chitosan. As sensor 5, based on CNT does not show linear response 


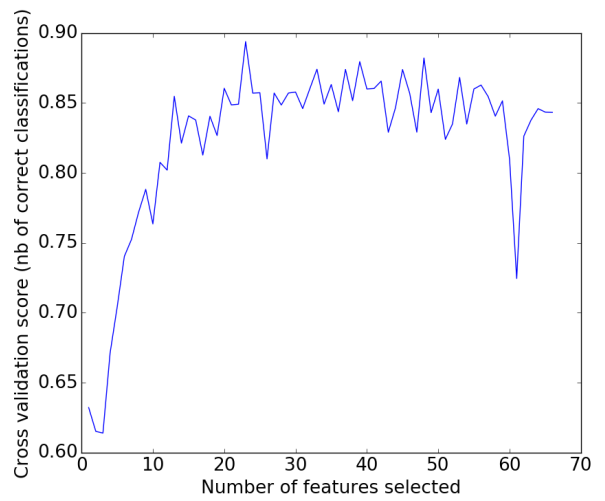

Fig. 4. Accuracy versus number of features selected by RFE for MLP algorithm.

in the low concentration range its coefficient $R^{2}$ is lower, 0.76 . Finally, repeatability of the sensors, characterized by standard deviation, varies from one sensor to another. Standard deviation is higher for chitosan sensors than for $\mathrm{TiO}_{2}$ or CNT. The high sensitivity to humidity makes chitosan based sensors less reliable. Standard deviation of $\mathrm{TiO}_{2}$ sensors are still high because the time of exposure is low, only $60 \mathrm{~s}$. A longer time of exposure improves the repeatability. For a classifier based on a regression model of one of these sensors, diagnostic errors are important. In these cases, the diagnostic accuracy is $41 \%$ for sensor $1,44 \%$ for sensor 2 based on chitosan and $44 \%$ for sensor 4, based on $\mathrm{TiO}_{2}$. The following work consists in crossing sensor responses and selecting the best features to see if the accuracy of diagnostic increases.

\section{B. Accuracy improvement by feature selection}

Figure 4 shows that the maximum accuracy is not obtained with the all available features. In fact, only a small part of them bring the best result: 13 features for LDA, 24 for MLP, 19 for RF and 39 for SVM.

TABLE I

DIAGNOSTIC ACCURACY (IN \%) OBTAINED BY DIFFERENT CLASSIFICATION ALGORITHMS AND DIFFERENT SET OF FEATURES AND WITH RFE.

\begin{tabular}{|c||c|c|c|c|c|}
\hline \multicolumn{1}{|c||}{} & \multicolumn{5}{c|}{$\begin{array}{c}\text { Features of the resistive curve } \\
\text { used to test the algorithm }\end{array}$} \\
\hline Tested Algorithm & $\Delta_{R}$ & $\delta_{R}$ & $\int_{R}$ & $\begin{array}{c}\Delta_{R}+\delta_{R} \\
+\int_{R}+\Delta_{R}\end{array}$ & RFE selected \\
\hline LDA & 64 & 64 & 67 & 80 & 84 \\
\hline RF & 77 & 77 & 50 & 80 & 83 \\
\hline SVM & 72 & 77 & 75 & 85 & 91 \\
\hline MLP & 75 & 78 & 63 & 85 & 88 \\
\hline
\end{tabular}

Figure 5 shows that the most important features are extracted from sensors 4 and 5 for MLP. However, these sensors do not bring enough information to accurately discriminate the samples (classification accuracy is $75 \%$ with these two sensors). According to the classifier, the best features are not the same. It is noteworthy that sensor 5 provides the least

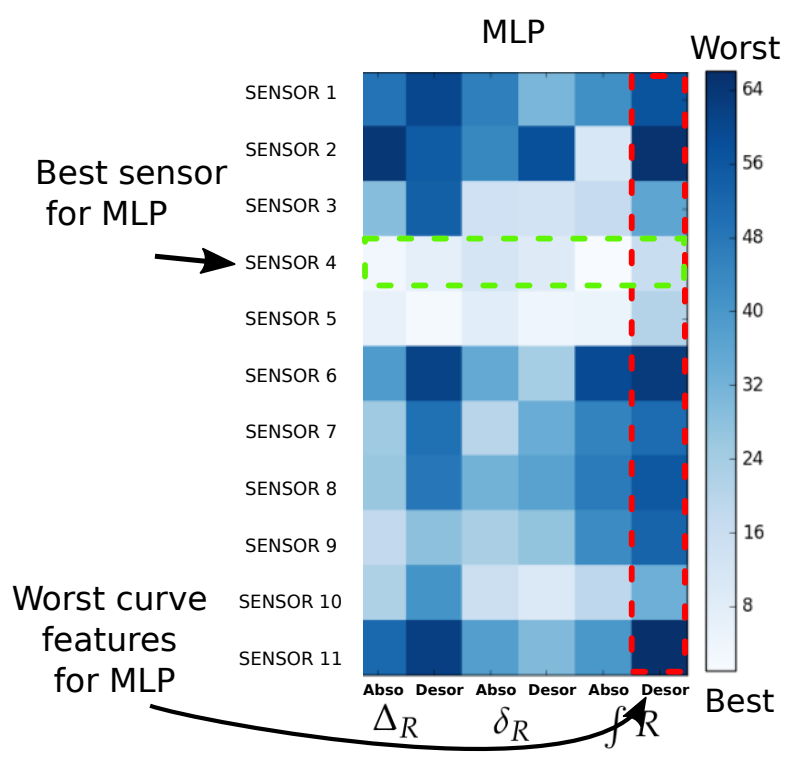

Fig. 5. Illustration of the RFE results for MLP algorithm.The best features are lighter.

information for LDA and the most information for MLP. This is in the sense that MLP can handle non-linear data in the contrast to LDA. Sensor 5, based on CNT, is the one with the worst linearity ( $R^{2}$ coefficient is 0.74$)$. However, no significant differences between the features extracted during absorption or desorption are evident from these results. Only the integral features during the absorption phase are useless for MLP and LDA, but not RF. This points out that testing several algorithms is an important step to select the right sensor array. Crossing the information of Figures 4 and 5 yields the best subset. The classification accuracy improvements brought in by the RFE algorithm are presented in Table I. With RFE, accuracy increases up to $91 \%$ for SVM, $87 \%$ for MLP and 84 $\%$ for LDA while reducing the set of necessary sensors down to 8,6 and 4 respectively. These values become interesting for a medical application.

\section{Conclusions}

This paper showed the advantages of the use of an electronic nose to detect ammonia in a breath simulation. Reliability of a single sensor system is impaired by problems with drift, sensor failure or repeatability. The use of a network of polyaniline sensors was made possible by the combination of the good sensitivity of polyaniline with ammonia while having differentiated responses thanks to different formulations. Thus, classification accuracy of $91 \%$ was achieved using SVM algorithm and feature selection algorithm compared to the 44 $\%$ obtained by basing the classification model on the linear regression of a sensor. As electronic noses can be embedded in a handheld device, these results pave the way for a portable diagnostic system for kidney diseases. 


\section{REFERENCES}

[1] M. Phillips, J. Herrera, S. Krishnan, M. Zain, J. Greenberg, and R. N. Cataneo, "Variation in volatile organic compounds in the breath of normal humans," Journal of Chromatography B: Biomedical Sciences and Applications, vol. 729, no. 12, pp. 75-88, Jun. 1999. [Online]. Available: http://www.sciencedirect.com/science/article/pii/S0378434799001279

[2] H. Haick, Y. Y. Broza, P. Mochalski, V. Ruzsanyi, and A. Amann, "Assessment, origin, and implementation of breath volatile cancer markers," Chem. Soc. Rev., vol. 43, no. 5, pp. 1423-1449, 2014. [Online]. Available: http://xlink.rsc.org/?DOI=C3CS60329F

[3] B. Grabowska-Polanowska, J. Faber, M. Skowron, P. Miarka, A. Pietrzycka, I. Sliwka, and A. Amann, "Detection of potential chronic kidney disease markers in breath using gas chromatography with mass-spectral detection coupled with thermal desorption method," Journal of Chromatography. A, vol. 1301, pp. 179-189, Aug. 2013.

[4] S. Davies, P. Spanel, and D. Smith, "Quantitative analysis of ammonia on the breath of patients in end-stage renal failure," Kidney International, vol. 52, no. 1, pp. 223-228, Jul. 1997.

[5] C. Turner, P. panl, and D. Smith, "A longitudinal study of ammonia, acetone and propanol in the exhaled breath of 30 subjects using selected ion flow tube mass spectrometry, SIFT-MS," Physiological Measurement, vol. 27, no. 4, p. 321, 2006. [Online]. Available: http://stacks.iop.org/0967-3334/27/i=4/a=001

[6] H. Haick, "Chemical sensors based on molecularly modified metallic nanoparticles," ResearchGate, vol. 40, no. 23, pp. 7173-7186, Dec. 2007.

[7] J.-1. L. Wojkiewicz, N. Redon, A. Pud, S. Mikhaylov, N. Ogurtsov, Y. Noskov, C. Collard, and W. Li, "Hybrid and Bio Nanocomposites for Ultrasensitive Ammonia Sensors," Proceedings, vol. 1, no. 4, p. 407, Aug. 2017. [Online]. Available: http://www.mdpi.com/2504$3900 / 1 / 4 / 407$

[8] S. Mikhaylov, N. A. Ogurtsov, N. Redon, P. Coddeville, J.-L. Wojkiewicz, and A. A. Pud, "The pani-dbsa content and dispersing solvent as influencing parameters in sensing performances of tio 2 hybrid nanocomposites to ammonia," RSC Adv., vol. 6, no. 86, pp. 82625$82634,2016$.

[9] K. Yan and D. Zhang, "Feature selection and analysis on correlated gas sensor data with recursive feature elimination," Sensors and Actuators B: Chemical, vol. 212, pp. 353-363, Jun. 2015.

[10] J. H. Leopold, L. D. J. Bos, P. J. Sterk, M. J. Schultz, N. Fens, I. Horvath A. Bikov, P. Montuschi, C. Di Natale, D. H. Yates, and A. Abu-Hanna, "Comparison of classification methods in breath analysis by electronic nose," Journal of Breath Research, vol. 9, no. 4, p. 046002, Dec. 2015.

[11] T. Hibbard, K. Crowley, and A. J. Killard, "Direct measurement of ammonia in simulated human breath using an inkjetprinted polyaniline nanoparticle sensor," Analytica Chimica Acta, vol. 779, pp. 56-63, May 2013. [Online]. Available: http://linkinghub.elsevier.com/retrieve/pii/S000326701300425X 